\title{
Zur Identität von Mathematiklernenden im schülerzentrierten Unterricht
}

\author{
Anne Güç • David Kollosche $\mathbb{D}$
}

Eingegangen: 15. November 2019 / Angenommen: 29. Juni 2021 / Online publiziert: 6. August 2021

(C) Der/die Autor(en) 2021

Zusammenfassung Identität von Mathematiklernenden beschreibt das Sprechen und Denken über das Selbst in Bezug zur Mathematik und kann das Mathematiklernen von Schülern und Schülerinnen und ihre Beziehung zum Fach maßgeblich beeinflussen. Frühere Studien zeigten für lehrerzentrierten Mathematikunterricht, dass die dem Mathematiklernen zugeschriebene Relevanz einen verkürzten und widersprüchlichen Diskurs darstellt, dass Lehrererklärungen Möglichkeiten des Verstehens monopolisieren und dass oft kaum Möglichkeiten gesehen werden, seine Persönlichkeit im Unterricht einzubringen. Diese Barrieren stehen einer Teilhabe am Mathematikunterricht für viele Schüler und Schülerinnen im Weg. Andere Studien wiesen jedoch daraufhin, dass schülerzentrierter Mathematikunterricht diese Hürden abbauen kann. In der hier berichteten Interviewstudie wurden Schüler und Schülerinnen aus zwei schülerzentriert unterrichteten Klassen der Sekundarstufe befragt, von der eine nach einem Freiarbeitskonzept arbeitete. Die Ergebnisse wurden entlang des poststrukturalistischen Konzepts der Identitätsarbeit ausgewertet. Zum einen zeigte sich selbst für die schülerzentriert unterrichteten Klassen ein verkürzter Relevanzdiskurs, der jedoch durch geeignete Nachfragen aufgebrochen werden konnte. Zum anderen bestätigten sich bezüglich der Rolle der Lehrererklärungen und der Persönlichkeitseinbringungen Vorteile für die Identitätsarbeit im schülerzentrierten Mathematikunterricht.

\footnotetext{
Anne Güç

Schule am Schloss Berlin, Schloßstraße 55a, 14059 Berlin, Deutschland

E-Mail: anne.guec@gmail.com

David Kollosche $(\bowtie)$

Institut für Didaktik der Mathematik, Universität Klagenfurt, Sterneckstraße 15, 9020 Klagenfurt am Wörthersee, Österreich

E-Mail: david.kollosche@aau.at
} 
Schlüsselwörter Identität von Mathematiklernenden · Identitätsarbeit ·

Schülerzentrierter Unterricht · Relevanz von Mathematiklernen ·

Lehrererklärungen · Persönlichkeitseinbringung

\section{Mathematics Learner Identity in Student-Centred Teaching}

Abstract Mathematics learner identity describes the way in which students talk and think about the self in relation to mathematics. They can significantly influence students' learning and their relationship to the subject. For teacher-centred mathematics teaching, previous studies showed that the relevance attributed to mathematics learning is a truncated and contradictory discourse, that teacher explanations monopolise possibilities of understanding, and that often few opportunities are seen to bring one's personality into the classroom. These barriers stand in the way of participation in mathematics education for many students. Other studies, however, indicated that student-centred mathematics teaching can reduce these barriers. In the interview study reported here, students from two student-centred classes in secondary education were interviewed. One of the classes used individualised instruction. The results were evaluated along the poststructuralist concept of identity work. On the one hand, even for the student-centred classes, a truncated discourse of relevance emerged, which could, however, be broken up by appropriate follow-up questions. On the other hand, advantages for identity work in student-centred mathematics lessons were confirmed with regard to the role of teacher explanations and personal contributions.

Keywords Mathematics learner identity $\cdot$ Identity work $\cdot$ Student-centred teaching $\cdot$ Relevance of mathematics education $\cdot$ Teacher explanations $\cdot$ Personal contributions

\section{Einleitung}

Identität von Mathematiklernenden (engl. mathematics learner identity, im Folgenden oft vereinfachend ,Identität“ genannt) beschreibt einen theoretischen und empirischen Zugang zum Verhältnis zwischen Mathematiklernenden einerseits und Fach und Unterricht andererseits. ${ }^{1}$ Forschung zu Identität baut auf einer soziologischen Rahmung auf, nutzt vorwiegend qualitative und explorative Methoden, und interessiert sich nicht nur für Zusammenhänge mit Lernerfolgen, sondern auch für ein Verständnis des sozialen Zustandekommens von Identität und für soziopolitische Folgen bestimmter Ausprägungen von Identität (Graven und Heyd-Metzuyanim 2019). Beispielsweise wird auf dieser Grundlage eine lernhinderliche Identität nicht mehr

\footnotetext{
1 Wir sprechen im Folgenden schlicht von „Mathematik“, da aus der Sicht der Schüler und Schülerinnen das, was Mathematik ausmacht, vornehmlich durch den Mathematikunterricht charakterisiert und eine Unterscheidung zwischen Schulmathematik und Universitätsmathematik oder anderen Formen von Mathematik nicht geläufig ist. Dass Schüler und Schülerinnen jedoch zwischen Mathematik als Sammlung von Lerninhalten und Mathematik als konkret umgesetztes Unterrichtsfach unterscheiden können, ist uns bewusst und wurde bei der Auswertung berücksichtigt.
} 
primär als individuelles Defizit aufgefasst, das man durch Interventionen auffangen sollte, sondern als sozial zu verstehendes Phänomen, in diesem Rahmen gemeinhin als aus Sicht der Lernenden durchaus sinnvolle Positionierung, und im kritischen Fall sogar als Konsequenz eines Mathematikunterrichts, der systematisch exkludierende Identitäten hervorbringt (z. B. Mendick 2006).

Die im Identitätsparadigma gewonnenen Erkenntnisse - Graven und Heyd-Metzuyanim (2019, S. 369-370) bieten eine kurze Übersicht - betreffen das Zusammenspiel zwischen der Identität von Mathematiklernenden und didaktischen Variablen wie Unterrichtsform und Prüfungsformaten, das Zusammenspiel zwischen Persönlichkeit und Identität, und hier im speziellen der Einfluss der Zugehörigkeit zu sozial abgegrenzten Gruppen auf der Grundlage von Hautfarbe, Ethnie, Gender und Behinderung. Wie im folgenden Theorieteil dargelegt wird, wurde in explorativen Studien insbesondere aufgezeigt, dass Schüler und Schülerinnen meist nur fragile Diskurse zur Relevanz von Mathematiklernen für die eigene Person nutzen, dass sich Schüler und Schülerinnen oft als passive Empfänger von Lehrererklärungen und nicht als eigenverantwortliche Lernende verstehen und dass Schülern und Schülerinnen im Mathematikunterricht oft Möglichkeit zur Einbringung der eigenen Persönlichkeit fehlen. In allen drei Punkten weisen Studien daraufhin, dass die Ausprägung der jeweiligen Identität von der Unterrichtsform abhängen kann. Um die Variabilität der Identität von Mathematiklernenden im Zusammenspiel mit der Unterrichtsform näher zu untersuchen und um frühere Forschungsergebnisse zu verifizieren, fokussierte die vorliegende Studie auf die Untersuchung der soeben genannten Aspekte von Identität in einer Freiarbeitsklasse und in einer Klasse mit schülerzentrierten Arbeitsformen.

\section{Theoretischer Rahmen}

\subsection{Identität von Mathematiklernenden}

Während Forschung zu Identität stets davon ausgeht, dass der soziale Kontext konstitutiv für ebendiese ist, liegen konkreten Studien durchaus unterschiedliche Definitionen von Identität und unterschiedliche Theorietraditionen zugrunde. Darragh (2016) arbeitete diese Unterschiede heraus und zeigte auf, dass die Frage, inwieweit diese unterschiedlichen Definitionen zu einem konsistenten Konzept von Identität vereinbar sind, hochkomplex und noch weitgehend ungeklärt ist. Radovic, Black, Williams und Salas (2018) zeigen darüber hinaus, dass sich die Hintergrundtheorien, auf die in einzelnen Studien Bezug genommen wird, durchaus überlappen, und Unterschiede vielmehr in der methodischen Umsetzung von Identitätsforschung zu finden sind. Während beispielsweise die meisten Studien von Selbstauskünften von Schülern und Schülerinnen auf der Grundlage von Interviews ausgehen, betonen andere Studien die Relevanz von Auskünften Dritter oder von Unterrichtsbeobachtungen.

Die vorliegende Studie basiert auf einem poststrukturalistischen Verständnis von Identität, welches auf Arbeiten von Foucault (1969/1981) und von Laclau und Mouffe (1985) zurückgeht und an anderer Stelle ausführlich vorgestellt wurde (Chronaki 
und Kollosche 2019). Foucault (1969/1981) und Laclau und Mouffe (1985) nutzten das Konzept des Diskurses, der bei ihnen die sprachliche und damit sozial vermittelte Praxis des Beschreibens und Konstituierens eines Gegenstandsbereichs bedeutet. Sozial vermittelt sind Diskurse insofern, als dass das in einem Diskurs sinnvoll Sagbare zwischenmenschlich bestimmt ist, auch wenn die individuelle Praxis dazu beitragen kann, dass die Grenzen dieser Bestimmung verschoben werden. Diskurse gelten als Praktiken des Beschreibens und Konstituierens, weil Diskurse nicht nur Realität abbilden, sondern selbst ordnen, formen und hervorbringen, wie Foucault (1975) unter anderem am Beispiel unseres Denkens über Delinquenz aufzeigte. An diesem Beispiel wird auch klar, dass Diskurse miteinander konkurrieren können (etwa solche, die Delinquenz als individuellen Defekt ansehen, mit solchen, die Delinquenz als Konsequenz eines entsprechenden gesellschaftlichen Milieus verstehen) und dass Diskurse zu unterschiedlichen Gegenstandsbereichen miteinander verbunden werden können (etwa solche zur Delinquenz mit jenen zu religiöser Sittlichkeit). Für Foucault (1969/1981) bestand die Aufgabe von Wissenschaft nun darin, das Wirken vorherrschender Diskurse zu verstehen und zu hinterfragen:

Eine Aufgabe, die darin besteht, nicht - nicht mehr - die Diskurse als Gesamtheiten von Zeichen (von bedeutungstragenden Elementen, die auf Inhalte oder Repräsentationen verweisen), sondern als Praktiken zu behandeln, die systematisch die Gegenstände bilden, von denen sie sprechen. Zwar bestehen diese Diskurse aus Zeichen; aber sie benutzen diese Zeichen für mehr als nur zur Bezeichnung der Sachen. Dieses mehr macht sie irreduzibel auf das Sprechen und die Sprache. Dieses mehr muß man ans Licht bringen und beschreiben. (S. 74)

Das Beleuchten der Wirkungsweisen von Diskursen ist für Foucault (1975) zentral, da Diskurse unsere Realität eben nicht nur ausdrücken, sondern formen: Diskurse generieren Wissen (so auch die Diskurse der Mathematik), sie rechtfertigen Praktiken (etwa jene des mathematischen Problemlösens) und sie legitimieren Institutionen (etwa den Mathematikunterricht selbst).

Identität lässt sich dann verstehen als Diskurs über das Selbst. Zum einen beschreibt dieser Diskurs nicht nur das Selbst, sondern konstituiert es erst als bedeutsamen Gegenstand. Zum anderen ist diese Beschreibung und Konstituierung sozial vermittelt, findet statt in einem Feld von konkurrierenden Diskursen und bildet Bezüge zu zahlreichen anderen Diskursen. Auf dieser Grundlage stellt sich mit Foucault (1969/1981) die Frage, in welcher Weise, unter welchen Umständen und mit welchen Wirkungen dieser Diskurs das Selbst hervorbringt. In unserem Fall bieten die Analyse unterschiedlicher Diskurse über das Selbst und Mathematik sowie die Untersuchung der vielfältigen Verbindungen zwischen diesen Diskursen einen Zugang zur sozial-diskursiven Konstituierung der Lernenden in Bezug zum Mathematikunterricht.

Laclau und Mouffe (1985) bauten Foucaults Diskurskonzept politikwissenschaftlich aus und betonten, dass das Individuum seine Identität in einem nie ruhenden Prozess des Sich-Verortens in einem Feld unterschiedlicher und miteinander streitender Diskurse ausprägt. Für Laclau und Mouffe sind Diskurse selbst immer nur vorläufige und nie endgültige Beschreibungen, die fortwährend verdrängt und verteidigt und dabei weiterentwickelt und ausgetauscht werden. Die Idee der Unabge- 
schlossenheit von Identität war schon im Werk von Foucault (1969/1981) angelegt. In ihrer Adaption von Foucaults Theorie zur Untersuchung der Identität von Mathematiklernenden in Zusammenspiel mit Genderidentität sprach Mendick (2005) allgemein von Identitätsarbeit (engl. identity work), um den unabgeschlossenen und performativen Charakter von Identität zu betonen.

Mendicks (2005, 2006) Untersuchung von Identitätsarbeit von Schülern und Schülerinnen in Bezug auf ihre Identität als Mathematiklernende gilt als richtungsweisend, da diese erstmals auf eine Sichtbarmachung der diskursiven Konstituierung von Identität in Interviewdaten abzielte. Die vorgestellte Perspektive hat Auswirkungen auf die Beforschung der Identität von Mathematiklernenden. Befragungssituationen rufen keine stabilen Identitäten ab und halten diese fest, sondern stellen selbst Situationen dar, in denen Diskurse in den Raum geführt werden, diesbezügliche Positionierungen vorgenommen werden und Identitätsarbeit stattfindet. Chronaki und Kollosche (2019) berichteten von einem Fall, bei dem eine solche Identitätsarbeit im Rahmen eines einzigen Schülerinterviews sichtbar wird.

\section{Forschungsstand}

Identität von Mathematiklernenden wurde aus vielen Perspektiven untersucht, die hier nicht in Gänze vorgestellt werden können. Eine wichtige, hier nicht weiter verfolgte Perspektive stellt der Einfluss der Zugehörigkeit zu sozialen Gruppen auf Identität dar. Paradigmatisch ist hier die Arbeit von Mendick (2006), die die Zusammenhänge zwischen Genderidentität und Identität von Mathematiklernenden untersuchte. Dazu befragte Mendick 42 16- bis 19-jährige englische Schüler und Schülerinnen in halbstrukturierten Interviews und beobachtete ihren Unterricht. Sie stellte unter anderem fest, dass unabhängig von tatsächlichen Leistungen mathematischer Erfolg gemeinhin mit Männlichkeit assoziiert wird und dass es infolge dieses vorherrschenden Diskurses Frauen deutlich schwerer als Männern fällt, eine lernförderliche Identität zu entwickeln. Mendicks Ergebnisse belegen, dass es wichtig ist, Identitätsarbeit vor dem Hintergrund widersprüchlicher Diskurse in den Blick zu nehmen, um Teilhabe zu erklären.

Ebenso richtungsweisend ist die Arbeit von Boaler und Greeno (2000), die die Identitäten von insgesamt 48 Schülern und Schülerinnen aus verschiedenen Analysis-Kursen in Kalifornien ebenfalls in halbstrukturierten Interviews untersuchten. Sie stellten heraus, dass die Schüler und Schülerinnen, die an einem offeneren, schülerzentrierten Mathematikunterricht teilnahmen, über dem Mathematikunterricht zugewandtere Identitäten verfügten und auch das Verhältnis zu ihrer Lehrkraft besser war. Außerdem waren diese Schüler und Schülerinnen zufriedener mit dem Mathematikunterricht und gaben an, ihre Persönlichkeit und eigene Lösungsideen mit einbringen zu können, während die Schüler und Schülerinnen, die frontal unterrichtet wurden, bemängelten, dass ihnen ebendiese Persönlichkeitseinbringung fehle und sie deshalb die Mathematik eher ablehnen. Die Studie unterstreicht, dass Identität als bestimmender Faktor für Teilhabe am Mathematikunterricht angesehen werden sollte. 
Wir werden unsere Diskussion des Forschungsstandes im Folgenden auf Zusammenhänge zwischen Identität einerseits und Relevanzzuschreibungen, Lehrererklärungen und Persönlichkeitseinbringung andererseits beschränken, da diese Aspekte in diesem Beitrag im Fokus stehen. Auf dieser Grundlage werden dann die Forschungsfragen formuliert.

\subsection{Relevanz von Mathematik}

Einen besonderen Fokus legen wir auf die Frage, inwiefern Schüler und Schülerinnen ihrem Mathematiklernen eine persönliche Relevanz zuschreiben. Im Rahmen unserer theoretischen Verortung sprechen wir dann von einer Relevanzzuschreibung, wenn Aspekte seines Diskurses über Mathematik es einem Individuum erlauben, Mathematik in seinem Diskurs über das Selbst axiologisch zu verorten. In anderen Worten geht es um das Artikulieren der Bedeutung von Mathematik für eigene Bestrebungen. Für eine Schülerin, die ihre Fähigkeit zum sicheren Argumentieren als zentralen Aspekt ihrer Identität ansieht, mag die Mathematik beispielsweise reizvoll werden, sobald der Diskurs über Mathematik das Fach als ein Gebiet des sicheren Schlussfolgerns ausweist (vgl. de Freitas 2008).

Ingram (2011) begleitete 31 neuseeländische Schüler und Schülerinnen über zwei Jahre bis zu ihrem zwölften Schuljahr und unterstrich in der Auswertung dieser Fallstudien, dass die Ausgeprägtheit von Relevanzzuschreibungen regelmäßig Auswirkungen auf Teilhabe, Erfolg und Wohlergehen im Mathematikunterricht hat. Zuvor hatten bereits Boaler et al. (2000) in ihrer Analyse der Identität von Mathematiklernenden auf der Grundlage von Interviews mit 120 14- bis 18-jährigen Schülern und Schülerinnen in England und den USA ebensolche Auswirkungen von Relevanzzuschreibungen dokumentiert und vorgeschlagen, dass Relevanzzuschreibungen Lernleistungen in vergleichbarem Maße beeinflussen wie kognitive Fähigkeiten. Besorgniserregend erscheint vor diesem Hintergrund, dass es Schülern und Schülerinnen offenbar oft schwerfällt, Relevanzzuschreibungen zu äußern, und dass geäußerte Relevanzzuschreibungen häufig fragil sind. In ihrer Auswertung der Interviews mit 112 deutschen Hauptschülern und -schülerinnen berichteten Maßß und Ege (2007), dass die Nützlichkeit von Mathematik zwar von der Mehrzahl der Befragten betont wurde, hierfür allerdings keine Beispiele genannt werden konnten, woraus die Autoren schlussfolgerten, dass die meisten Befragten ,keinen Einblick in die Bedeutung von Mathematik für die Gesellschaft haben“ (S. 73). Auch wenn Maaß und Ege ihre Daten nicht aus der Perspektive eines Identitätsparadigmas interpretierten und „Nützlichkeit“ sicherlich nur einen Teil möglicher Relevanzzuschreibungen ausmacht, zeichnete sich hier bereits ab, dass im vorherrschenden Diskurs zur Nützlichkeit von Mathematik allzu leicht Lücken aufbrechen, die für Relevanzzuschreibungen hinderlich werden können.

Obwohl explorative Studien überzeugende Hinweise auf die Bedeutung von Relevanzzuschreibungen für das Mathematiklernen liefern konnten, wurde die Natur solcher Relevanzzuschreibungen aus der Perspektive der Identitätsforschung nicht weiter untersucht. Eine Ausnahme bildet eine Studie von Kollosche (2017a), die die Resultate von Maaß und Ege (2007) bestätigt, über die Hauptschule hinaus verallgemeinert und im Identitätsparadigma interpretiert. Dazu wurden 23 Acht-, Neunt- und 
Zehntklässler öffentlicher Schulen unterschiedlicher Schulzweige in Berlin, Brandenburg und Sachsen-Anhalt, von denen nur zwei aus der gleichen Klasse und Schule kamen, in halbstrukturierten Interviews befragt. „Glaubst du, dass dein Mathematikunterricht für dein Leben wichtig ist oder sein wird?" war eine der Interviewfragen, die Äußerungen zur Relevanz von Mathematik und Mathematikunterricht provozierte. Nach Analyse der Schülerantworten stellte der Autor fest, dass sich Relevanzzuschreibungen in der Sekundarstufe in der Regel auf das inhaltliche Lernen von im Alltag nützlicher elementarer Mathematik im Sinne von Heymanns (1996) Katalog alltagsrelevanter Inhalte (S. 135f.) beschränken und weiterführende Bildungsziele wie beispielsweise die Charakterbildung oder der Aufbau prozessbezogenen Wissens und Könnens unbeachtet bleiben. Zu vergleichbaren Ergebnissen kamen Sachdeva und Eggen (2019) in einer Studie in Norwegen. Kollosche (2017a) vermutete, dass die Standardantwort der Schüler und Schülerinnen ein ideologischer Schutzmechanismus ist, der diese davor bewahrt, elaboriertere Antworten auf die Relevanzfrage zu finden. Er konnte zeigen, dass die Fragilität dieser Standardantwort ohne Zutun des Interviewenden bereits einigen Befragten im Laufe ihres Interviews klar wird. Unklar blieb jedoch, inwieweit die Eingeschränktheit der Diskurse der Befragten zur Relevanz von Mathematik ein Artefakt der Befragungssituation darstellte und ob etwa ein weiteres Nachfragen über diese fragile Standardantwort hinaus elaboriertere Erklärungen hätte provozieren können.

\subsection{Lehrererklärungen}

Boaler und Greeno (2000) konnten in ihrer obengenannten Studie aufzeigen, dass die Identität von Mathematiklernenden von der erlebten Unterrichtsform abhängen kann. So fiel es Lernenden gerade in einem von Lehrervorträgen und Einzelübungen geprägten Unterricht häufig schwer, die Tätigkeiten im Mathematikunterricht mit ihrem Selbstbild in Einklang zu bringen, während Lernende in einem auf Gruppenarbeit und Aushandlung aufbauenden Unterricht hier keine Schwierigkeiten zeigten und häufiger äußerten, sich selbst freiwillig mathematisch weiterbilden zu wollen. Als Erklärung diente hier, dass viele, wenn auch nicht alle, Lernenden sich selbst nicht als gehorsamen Imitator, sondern als kreativen Gestalter sehen wollten, was mit dem lehrerzentrierten Unterricht für viele nicht vereinbar war.

Andersson et al. (2015) berichteten ausführlich von zwei schwedischen Schülerinnen der Sekundarstufe II, die sich selbst als „maths anxious“ und „maths hater“ (S. 145) beschrieben und lehrerzentrierten Unterricht erlebt hatten. Während einer Projektwoche im Mathematikunterricht wurden sie aber zu interessierten und engagierten Teilnehmerinnen und sie begannen, ihre ursprünglichen Identitäten zu hinterfragen. Dieses Beispiel zeigt nicht nur erneut und dieses Mal am Beispiel von Projektunterricht, wie Unterrichtsformen Identitäten beeinflussen können, sondern bietet auch eine Dokumentation von Identitätsarbeit im Vollzug.

Auf der Grundlage der Daten aus seiner obengenannten Studie konnten ähnliche Ergebnisse in den untersuchten deutschen Schulen reproduzieren werden (Kollosche 2017b). Dabei nutzte Kollosche den Begriff der Lehrererklärung, die er als ,,verbal and embodied communication of knowledge and skills“ verstand (S. 1505). Lehrerzentrierte Unterrichtsformen, die von Boaler und Greeno (2000) und von Andersson 
et al. (2015) als problematisch für die Ausbildung einer lernförderlichen Identität präsentiert wurden, sind nun dadurch charakterisiert, dass Erklärungen durch den Lehrer oder die Lehrerin hier der primäre oder gar der einzige bereitgestellte Zugang zu mathematischem Verständnis darstellen. Im Gegensatz dazu bieten die eigenaktiven und vielfältigen kommunikativen Anlässe in Gruppen- und Projektarbeit weitaus mehr Möglichkeiten für Verständnis. Dieser Umstand liegt nicht nur darin begründet, dass hier (quantitativ) mehr oder (qualitativ) passendere Erklärungen zur Verfügung stehen, sondern vor allem auch darin, dass diese vielfältigen Kommunikationsanlässe in höherem Maße eine produktive Teilhabe im kollektiven Verständnisprozess ermöglichen.

Kollosche (2017b) stellte fest, dass das Thema „Lehrererklärungen“ in den erhobenen Interviewtexten eine zentrale Stellung einnahm. So wurde das Thema von allen 23 Befragten aufgeworfen, obwohl keine Interviewfrage das Thema direkt adressierte. Zur Interpretation der Schülerdiskurse wurde außerdem darauf hingewiesen, dass die Befragten durchgängig von einem lehrerzentrierten Unterricht berichteten. Es zeigte sich zum einen, dass die Qualität der Lehrererklärungen in den meisten Fällen die Zufriedenheit mit der Lehrperson bestimmte. Aus der Identitätsperspektive besonders interessant war, dass die genutzten Diskurse unzureichende Lehrererklärungen als Erklärung für gute und schlechte Leistungen im Mathematikunterricht nutzten. Kollosche betonte, dass diese Organisation von Mathematikunterricht neben einer Etablierung von fachlichen Hierarchien auch vermeidbare Abhängigkeiten im Lernprozess erzeuge. In der Tat zeigten sich in den Interviews einige Befragte, die den Erklärungen ihrer Lehrperson nicht folgen können, hilflos, verärgert und verzweifelt. An Hand der Schüleräußerungen konnte nachgezeichnet werden, dass es den Lernenden unterschiedlich schwerfällt, als Zuhörer zu lernen, so dass Erfolg in einem derart organisierten Mathematikunterricht neben mathematischem Verständnis weitgehend ohne bildungspolitische Legitimation vor allem auch Konzentrationsvermögen prüfe. Er wies aber auch darauf hin, dass ein lehrerzentrierter Mathematikunterricht es Schülern und Schülerinnen überhaupt erst ermöglicht, eine eigenverantwortliche Rolle im Mathematiklernen von sich zu weisen und die Verantwortung für mathematisches Verständnis bei der Lehrperson zu lokalisieren.

\subsection{Persönlichkeitseinbringung}

Als wichtiger Einfluss auf die Identität als Mathematiklernende erwies sich die Ausgestaltung der Möglichkeiten, seine Persönlichkeit in den Mathematikunterricht einbringen zu können. Unter Persönlichkeitseinbringung im Mathematikunterricht verstehen wir die Praxis, individuell einzigartiges Wissen und Können zu den Diskursen des Mathematikunterrichts beizutragen. Eine solche Praxis erlaubt es dem Individuum, seine Identität weiterzuentwickeln und dabei auf den Mathematikunterricht Bezug zu nehmen. Mit ,individuell einzigartigem Wissen und Können“ sind dabei gerade nicht regelgeleitete Praktiken und dadurch gewonnene Erkenntnisse gemeint, auch wenn diese individuell durchgeführt und erlangt werden, sondern an die Person gebundene Erfahrungen, Ideen und Sichtweisen.

Boaler und Greeno (2000) berichteten, dass viele Befragte aus der untersuchten lehrerzentriert unterrichtenden Schule angaben, dass Mathematikunterricht aus dem 
Abarbeiten von vorgegebenen Regeln bestehe und mit dem Einzelnen nichts zu tun habe. Solche Diskurse über Mathematikunterricht erschwerten es vielen Schülern und Schülerinnen, sich selbst als wertvolle Akteure und Akteurinnen im Mathematikunterricht zu begreifen. Das Problem wurde in späteren Fallstudien noch näher beleuchtet (Andersson et al. 2015; Kollosche 2019a). Sowohl Boaler und Greeno (2000) als auch Andersson et al. (2015) konnten jedoch feststellen, dass fehlende Möglichkeiten der Persönlichkeitseinbringung in schülerzentrierten Lernumgebungen nicht beklagt werden. Boaler und Greeno (2000) stellten dies bei ihren Befragten aus der kontrastierend untersuchten, schülerzentriert unterrichtenden Schule fest; Anderson et al. (2015) konnten die Veränderung des Diskurses zweier Schülerinnen im Rahmen einer Projektarbeit festhalten. Diese Studien stützen die These, dass durch eine schülerzentrierte Gestaltung des Mathematikunterrichts Hürden zur Persönlichkeitseinbringung abgebaut werden können.

\subsection{Formulierung der Forschungsfragen}

Die bisherigen Ergebnisse zur von Schülern und Schülerinnen empfundenen Relevanz von Mathematik und Mathematiklernen deuten großen Handlungsbedarf an, werfen jedoch auch die Frage auf, ob sich Schüler und Schülerinnen in anders gestalteten Befragungssituationen nicht doch noch in weiteren Diskursen zur Relevanz verortet hätten. In der vorliegenden Interviewstudie folgte daher auf die Frage „Glaubst du, dass Mathe für dein Leben wichtig ist oder sein wird?“ die Frage „Lernst du in Mathe etwas für dich, das über die Lerninhalte hinausgeht?“. Die erste Forschungsfrage der vorliegenden Studie fragt dann, inwieweit sich die Schüler und Schülerinnen durch das gezielte Fragen nach Relevanzzuschreibungen jenseits des Lernens von Mathematik in weiteren Diskursen zur Relevanz von Mathematik verorten.

Die Ergebnisse von Kollosche (2017b) weisen darauf hin, dass die Qualität von Lehrererklärungen ein zentraler Diskurs für die Identitätsarbeit für Schüler und Schülerinnen im lehrerzentrierten Mathematikunterricht darstellt. Dies wird insbesondere dann problematisch, wenn Schüler und Schülerinnen sich in einem solchen Diskurs als passive Opfer von unzureichenden Erklärungen verstehen. Da weder Boaler und Greeno (2000), noch Andersson et al. (2015) die Schülerdiskurse zu Lehrererklärungen näher untersucht haben, ist jedoch unklar, inwieweit solche Diskurse durch schülerzentrierten Unterricht aufgebrochen werden können. Die zweite Forschungsfrage der vorliegenden Studie fragt daher, welche Rolle Diskurse zur Qualität von Lehrererklärungen in der Identitätsarbeit im schülerzentrierten Unterricht spielen.

Fehlende Möglichkeiten der Persönlichkeitseinbringung wurden in verschiedenen Studien als typische Hürde für eine positiv-produktive Positionierung des Selbst im Diskurs über den Mathematikunterricht identifiziert. Dieses Problem wurde bisher aber nur aus lehrerzentriertem Unterricht berichtet, wohingegen diese Hürden aus schülerzentriertem Unterricht nicht berichtet wurden. Die dritte Forschungsfrage der vorliegenden Studie fragt nun, wie die Befragten mit schülerzentriertem Unterricht ihre Möglichkeiten zur Persönlichkeitseinbringung bewerten. 


\section{Methodisches Vorgehen}

Im Folgenden wird erläutert, wie die Untersuchung der Forschungsfragen methodisch umgesetzt wurde. Dazu wird zunächst auf das in einer der beiden untersuchten Klassen umgesetzte Freiarbeitskonzept Mathematiika, dann auf die Methoden der Datenerhebung und schließlich auf die Methoden der Auswertung eingegangen.

\subsection{Mathematiika und Freiarbeit}

Das Konzept Mathematiika wurde 2016 von den beiden Mathematiklehrerkräften Lena Florian und Sebastian Grabow in Anlehnung an ein vergleichbares finnisches Modell, das von Pekka Peura konzipiert wurde, entwickelt (Florian und Grabow 2017). In dieser Unterrichtsform ist das Schulhalbjahr im Fach Mathematik in Module aufgeteilt, die den Schülern und Schülerinnen bereits zu Schuljahresanfang bekannt sind. Darüber hinaus wird offengelegt, welche Module prüfungsrelevant sind und wie viel Raum die Bearbeitung der einzelnen Module in etwa einnehmen sollte. Jedes Modul bietet verschiedene Zugänge zum Erwerb mathematischer Kompetenzen: In der Bibliothek werden ihnen zum Großteil Informationstexte und Beispielaufgaben zur Verfügung gestellt, wohingegen die Mediathek Erklär- und Beispielvideos zu jedem Thema bietet. Schließlich haben sie in der Safari noch die Möglichkeit, mit Hilfe von Lernumgebungen, die an das entdeckende Lernen angelehnt sind, die Inhalte selbständig zu entwickeln. Es ist ihnen möglich, jederzeit zwischen den verschiedenen Zugängen zu wechseln. Die Materialien werden den Schülern und Schülerinnen über eine Onlineplattform zur Verfügung gestellt. Im Unterricht werden den Schülern und Schülerinnen Tablets zur Verfügung gestellt. Während die Erarbeitung individuell erfolgt, arbeiten zum Üben alle mit dem gleichen Aufgabenpool. Am Ende eines jeden Moduls erbringen die Schüler und Schülerinnen eine zusätzliche Leistungsbeurteilung, deren Form und Gestaltung von ihnen selbst gewählt werden kann. Zusätzlich wird am Ende eines jeden Moduls ein Test geschrieben. Mit Hilfe einer kollaborativen Online-Tabelle können sich die Schüler und Schülerinnen zum Ende einer jeden Stunde selbst reflektieren und ihren Fortschritt so auch für die Lehrperson sichtbar machen. Durch die unterschiedlichen Zugänge und das Übungsmaterial ist es möglich, ein hohes Maß an Differenzierung in den Unterricht zu integrieren. Dabei werden die Schüler und Schülerinnen sich ihrer eigenen Stärken und Schwächen bewusst und müssen so selbst die Verantwortung für ihren eigenen Lernprozess übernehmen. So argumentieren auch die Entwickler dieser Unterrichtsform, dass durch das selbstständige Lernen nicht nur die mathematischen Fähigkeiten, sondern auch Kompetenzen wie die Selbstorganisation oder auch Selbstmotivation trainiert werden.

\subsection{Datenerhebung}

Als Erhebungsmethode wurde ein teilstandardisiertes (halboffenes) Leitfadeninterview nach Flick (2000) gewählt, also ein Interview, das sich an einem vorformulierten Interviewleitfaden orientierte. Dieser Interviewleitfaden umfasste 38 Fragen, von denen jedoch 13 Fragen nur optional gestellt wurden, in der Regel als Nachfragen, 
um bei nur umfangsarmen Antworten weiterführende Antworten zu provozieren. Flick betonte, dass es durch die Wahl der Erhebungsmethode möglich sei, nicht nur allen Befragten in etwa die gleichen Fragen zu stellen, sondern sich die Möglichkeit offenzuhalten, die Abfolge der Fragen zu ändern, Fragen wegzulassen, wenn diese bereits beantwortet wurden, und auf einzelne Aussagen direkter einzugehen als es in einem standardisierten Interview möglich gewesen wäre (S. 113). Die Schüler und Schülerinnen hatten durch die Methode außerdem die Möglichkeit, auf die Fragen frei zu antworten; sie konnten also auch entscheiden, wie umfangreich sie auf welche Frage antworten möchten. Insbesondere konnte durch die halboffenen Interviews sichergestellt werden, dass zu den jeweiligen Antworten auch Erklärungen geliefert wurden. Ein weiterer wichtiger Vorteil von teilstandardisierten Interviews liegt darin, dass es den Befragten ermöglicht wird, für sie relevante Themen zu adressieren. Flick argumentierte, dass durch die offene Gestaltung der teilstandardisierten Interviews die Möglichkeit eingeräumt werde, die Sichtweisen der Befragten besser zu erfassen als es in standardisierten Interviews oder Fragebögen möglich wäre (S. 94). Alle Befragungen wurden von der Erstautorin durchgeführt. Während der Befragung wurde großer Wert auf eine gemütliche und offene Atmosphäre sowie auf Empathie gelegt. Dies sollte dazu führen, dass die Schüler und Schülerinnen offen und nicht sozial erwünscht antworteten. Der eingesetzte Fragebogen ist im Anhang an diesen Beitrag zu finden.

Die Datenerhebung fand in zwei allgemeinen Leistungs- und Begabungsklassen einer weiterführenden Schule mit gymnasialer Oberstufe im Bundesland Brandenburg statt. Schüler und Schülerinnen dieser Klassen kamen anders als in Brandenburg sonst üblich bereits nach ihrem vierten Schuljahr an die Sekundarschule, gelangten in 12 statt 13 Jahren zum Abitur und wurden an Hand ihrer guten Grundschulnoten ausgewählt. Die Klassen waren jedoch keine speziell mathematischen Begabungsklassen und die mathematischen Fähigkeiten der Schüler und Schülerinnen variierten durchaus. Es handelte sich um eine 8. Klasse, welche mit dem MathematiikaKonzept im Mathematikunterricht arbeitete, und eine 9. Klasse, deren Lehrkraft die Inhalte und Lernmittel für ihre Schüler und Schülerinnen auswählt, diesen aber Freiheiten im sozialen Arrangement ihres Lernprozesses lässt. Damit wurden Daten aus einer Klasse mit einem Freiarbeitskonzept einschließlich spezieller Unterrichtsmaterialen sowie aus einer Klasse mit einem inhaltlich lehrergesteuerten, aber sozialmethodisch schülerzentrierten Unterrichtsgeschehen gewonnen. Es wurden aus der 8. Klasse alle 24 Schüler und Schülerinnen und aus der 9. Klasse alle 21 Schüler und Schülerinnen befragt. Die Durchführung des Interviews erfolgte im Sommer 2018 unter vier Augen in einem separaten Raum im Schulgebäude.

\subsection{Datenauswertung}

Die Interviews wurden aufgenommen und im Anschluss transkribiert. Allen Teilnehmern und Teilnehmerinnen der Studie wurden Pseudonyme zugeordnet. Dann wurde eine thematic analysis nach Braun und Clarke (2006) durchgeführt, um typische Diskurse von Identität als Mathematiklernende in den Schüleräußerungen zu identifizieren. In diesem induktiven Ansatz werden Aussagen zu bestimmten Diskursen in sogenannten Themen zusammengefasst, welche allgemein beschrieben 
und mit tatsächlichen Schüleräußerungen unterlegt werden. Waren Aussagen unklar, wurden sie mit Hilfe weiterer Aussagen zum Sachverhalt im Verlauf des Gesprächs interpretiert. Dennoch unklare sowie widersprüchliche Aussagen wurden nicht den Themen zugeordnet. Aussagen, die Ausnahmen enthielten, wurden unabhängig von ihren Ausnahmen codiert. Aussagen, die an Bedingungen geknüpft waren, wobei unklar war, ob diese erfüllt sind, wurden nicht berücksichtigt.

\section{Ergebnisse}

Im Rahmen der durchgeführten thematic analysis konnten in den Schülerinterviews 16 wiederkehrende Themen rekonstruiert werden. Wir beschränken unsere Diskussion der Ergebnisse im Folgenden jedoch auf für die Forschungsfragen relevante Themen und beleuchten, welche Diskurse vorgetragen wurden, wie diese mit anderen Diskursen verknüpft sind und wie die Diskurse die Identitätsarbeit der Schüler und Schülerinnen beeinflussten.

\subsection{Relevanz von Mathematik}

Die erste Forschungsfrage lautete, inwieweit sich die Schüler und Schülerinnen durch das gezielte Fragen nach Relevanzzuschreibungen jenseits des Lernens von Mathematik in weiteren Diskursen zur Relevanz von Mathematik verorten. Festzuhalten ist, dass die Befragten der vorliegende Studie auf der Grundlage der aus Vorstudien übernommenen Frage „Glaubst du, dass Mathe für dein Leben wichtig ist oder sein wird?" die in den früheren Studien berichteten Verweise auf das Lernen elementarmathematischer Inhalte reproduzieren. Die nachgestellte Frage „Lernst du in Mathe etwas für dich, das über die Lerninhalte hinausgeht?" provozierte die Befragten jedoch, ihre Relevanzzuschreibungen in einer Fülle anderer Diskurse zu positionieren. Bemerkenswert ist, dass sich die relative Häufigkeit, mit der dies gelang, zwischen der Freiarbeitsklasse und der anderen Klasse deutlich unterschied.

Auf die erste der im letzten Absatz genannten Interviewfragen antworteten $63 \%$ der Schüler und Schülerinnen aus Klasse 8, die nach dem Freiarbeitskonzept Mathematiika unterrichtet wurde, dass Mathematik im Alltag nützlich sei. In Klasse 9, die schülerzentrierte Lernformen nutze, taten dies $57 \%$ der Schüler und Schülerinnen. In beiden Klassen erklärte jeweils nur eine Person, dass Mathematik nicht nützlich sei. Jedoch waren lediglich zwei Schüler und Schülerinnen der Klasse 8 und niemand aus Klasse 9 auch auf Nachfragen in der Lage, konkrete Beispiele nützlicher Inhalte aus dem weiterführenden Mathematikunterricht $\mathrm{zu}$ nennen. In beiden Fällen handelt es sich um die Winkelberechnung: Nathan verwies auf Berechnungen beim familiären Hausbau und Ricky auf die Nutzung von Winkelberechnung beim Feldhockey in ihrer Freizeit.

Einige Befragte nannten, wenn sie nach einem Beispiel für nützliche Mathematik gefragt wurden, elementarmathematische Inhalte. Acht Schülern und Schülerinnen aus Klasse 8 und fünf Schülern und Schülerinnen aus Klasse 9 fiel nur das Beispiel Einkaufen ein. Deutlich wurde das bei Anita aus der Klasse 8. Auf die Frage, mit welchen Wörtern sie die Mathematik beschreiben würde, antwortet sie „,wichtig“ und 
„man braucht es im Alltag“. Später fügt sie noch hinzu, dass „,man [...] es halt später [braucht]", ihr fiel aber bis auf das Einkaufen keine Alltagssituation ein, in der sie mathematisches Wissen benötigt. Lediglich neun Schüler und Schülerinnen je Klasse können mehrere Beispiele für Alltagssituationen (beim Kochen, zur Erstellung der Steuererklärung, zum Renovieren, beim Lesen des Fahrplans und zur Berechnung der Wartezeit oder zur Gartenplanung) nennen, in denen sie Mathematik benötigen. Emin aus Klasse 9 erklärt: „Mathematik findet man halt überall im Alltag, du kannst damit einfach alles berechnen [...]". Auf die Frage, ob Mathematik im Alltag wichtig sei, antwortet er: „Man kennt diese klassischen Situationen: Einkaufsladen, wenn man was nachrechnen muss, oder [...] wenn man sein Haus verwaltet, Garten plant“. Emin verknüpft den Diskurs zur Relevanz von Mathematik also mit einer Reihe von Aktivitäten, in denen elementares mathematisches Wissen nützlich ist. Hingegen konnten aus der Klasse 8 sechs und aus der Klasse 9 fünf Schüler und Schülerinnen überhaupt kein Beispiel dafür angeben, wofür man Mathematik im Alltag brauche.

Eine hohe Nützlichkeit schrieb der Mathematik ein Großteil der Schüler und Schülerinnen zu, die der Mathematik sonst eher mit einer Abneigung gegenüberstehen. Beispielhaft sei an dieser Stelle Charlotte aus Klasse 8 zu nennen. Auf die Frage hin, welche Gedanken ihr bei Mathematik als erstes in den Kopf kommen, nennt Charlotte ,langweilig, dass ich es nicht so gut verstehe, keine guten Gedanken“ und sie fügt hinzu, dass sie an Mathematik eigentlich nicht viel gut finde, eigentlich möge sie alles nicht. Dennoch ist Charlotte eine der wenigen Personen, die konkrete Alltagssituationen, in der man Mathematik benötige, nennt, etwa die Steuererklärung oder auch das Kochen. Sie schreibt der Mathematik also trotz der negativen Attitüde eine hohe Nützlichkeit zu. Ähnlich geht es auch Milo aus Klasse 9. Er beschreibt Mathematik als ,langweilig“ und „Stress“, schreibt der Mathematik hohe Anstrengungen zu, verweist aber nicht nur die Notwendigkeit der Mathematik in Alltagssituationen wie dem Hausbau, sondern äußert auch, dass Mathematik eigentlich in allen Berufen enthalten und somit unumgänglich sei. ${ }^{2}$

Insgesamt verweist die von allen geteilte Aussage der Befragten, dass es im Mathematikunterricht um das Erlernen von praktisch nützlichen Inhalten gehe, und die darauf folgende Schwierigkeit oder Unmöglichkeit, hierfür Beispiele zu nennen, darauf, dass die Befragten einen dominanten Diskurs reproduzierten, den sie dann aber kaum mit ihren konkreten Erfahrungen in Einklang bringen konnten. Im Zusammenhang mit der Beispielfindung räumte beispielsweise Anita ein: „Mathe braucht man ja eigentlich bestimmt überall und wird ja eigentlich auch irgendwie immer so gesagt, dass Mathe sehr wichtig ist und deswegen denk ich das auch mal so“. Ein weiteres Beispiel ist Ilyas aus Klasse 9, der argumentierte, dass Mathematik ein Fach sei,

das man ziemlich ernst nehmen sollte, [weil es] eigentlich immer im Alltag wichtig ist und auch, obwohl man sich so oft die Frage stellt „Wozu brauch man jetzt das und wozu das?“،, aber ich bin mir ziemlich sicher, dass man min-

\footnotetext{
2 Einem der anonymen Gutachtenden dieses Beitrags kam hier der Gedanke, dass gerade Schüler und Schülerinnen, die von sich aus wenig Motivation für Mathematik zeigen, von Betreuungspersonen zu Motivationszwecken auf eine große Nützlichkeit der Mathematik hingewiesen werden. Das würde erklären, warum gerade auch diese Schüler und Schülerinnen entsprechende Positionen vertreten.
} 
destens $60 \%$ von allem, was man bisher gemacht hat, bestimmt irgendwann mal braucht.

Ilyas fand Mathematik nicht nur sehr wichtig; diese Relevanz löste in ihm, wie er ferner berichtete, auch einen großen Druck aus, wenn er eine Klassenarbeit schreiben musste. Auch später betonte er noch einmal die Notwendigkeit der Mathematik und sagte, dass ,Mathe eigentlich immer auch im Alltag wichtig ist, weil [...] es gibt bestimmt tausend Alltagssituationen, wo man da irgendwas ausrechnen muss“. Doch obwohl er die Relevanz mehrfach betonte, fiel ihm kein Bespiel aus dem Alltagsleben ein, in dem Mathematik Anwendung findet.

Die nachgeschobene Frage ,Lernst du in Mathe etwas für dich, das über die Lerninhalte hinausgeht?“" eröffnete jedoch neue Diskurse für die Relevanzzuschreibungen der Befragten. Fast die Hälfte der Befragten gingen in ihrer Antwort auf diese Frage auf Kompetenzen wie selbstständiges Arbeiten, Selbstmotivation, Selbstorganisation, Sozialkompetenzen, Offenheit gegenüber anderen Lösungswegen, Selbstbewusstsein und Fehlertoleranz sowie auf das Trainieren des Denkvermögens, der logischen Denkfähigkeit und der Konzentrationsfähigkeit ein.

Auffällig sind dabei die Klassenunterschiede. In Klasse 8, die nach dem Mathematiika-Konzept lernte, gaben knapp $60 \%$ der Schüler und Schülerinnen und damit $30 \%$ mehr als in Klasse 9 an, dass sie im Mathematikunterricht etwas vermittelt bekommen, das über die Lerninhalte hinausgehe. Ricky sah eine klare Verbindung zwischen dem Erlernen entsprechender Fähigkeiten und dem Freiarbeitskonzept in ihrer Klasse:

Ja, also einfach, dass wir viel [...] selber Arbeiten und dass wir selbstständiger werden dadurch [...] weil im späteren Leben können wir uns ja auch nicht einfach auf die Arbeit setzen „Nee jetzt habe ich keine Lust, das mache ich nicht“, sondern müssen wir halt trotzdem, auch wenn wir keine Lust haben, weiter arbeiten und das ist ja jetzt in Mathematiika genauso.

Sie zog also eine Verbindung zwischen der durch Mathematiika erlernten Selbstständigkeit und der Nützlichkeit für das spätere Berufsleben. Auch Ursula bestätigte, dass man im Rahmen dieser Freiarbeitsmethode lerne, ,,selbstständig zu werden, zu arbeiten“. Sero fügte hinzu, dass genau dieses selbstständige Lernen für ihn zu Beginn mit großen Motivationsproblemen verbunden war, was dazu führte, dass er sich manchmal ganze Unterrichtsstunden nicht mit mathematischen Inhalten auseinandersetzen konnte. Erst mit der Zeit schaffte er es, sich selbst so zu motivieren und zu organisieren, dass er nun ,seine Aufgaben macht und sich nicht mehr so leicht ablenken lässt"“. Ein weiteres Beispiel ist Nathan. Auf die Nachfrage, ob dieser im Unterricht etwas lerne, das über die Lerninhalte hinausgehe, negierte dieser zunächst die Frage. Im Rahmen der Frage, welche Vorteile die Freiarbeitsmethode Mathematiika mit sich bringe, antwortete er dann aber, dass man sich gegenseitig helfen könne, wobei „die Sozialkompetenz [...] auch irgendwie gefördert“ werde.

Aber auch in Klasse 9 gab es Schüler und Schülerinnen, die über das inhaltliche Lernen hinausgehende Kompetenzen nennen, welche durch den Mathematikunterricht geschult werden. So antwortete Ilyas aus Klasse 9 auf die Frage, ob er im Matheunterricht etwas lerne, das über die Lerninhalte hinausgehe: 
Hm. Ich glaube, Mathe kann man allgemein so Selbstbewusstsein, sich selbst mehr aneignen und vielleicht auch ein bisschen mehr Gruppenkompetenzen und [...] auch mal nicht Angst zu haben, die Aufgabe falsch zu sagen oder sowas [...], weil wir sind halt da um zu lernen, nicht um alles perfekt zu können.

Auch Leon aus Klasse 9 bestätigte, dass er den Mathematikunterricht als sehr wichtig einschätze, denn Mathematik fördere nicht nur das Fachwissen, sondern „auch das Denkvermögen und die logische Denkfähigkeit“". Interessanterweise verneinte Leon jedoch die spätere Frage, ob er etwas im Mathematikunterricht lerne, das über die Lerninhalte hinausgehe. Ein letztes Beispiel ist Emilyan aus Klasse 9, der sagte, dass der Mathematikunterricht ganz besonders die Konzentrationsfähigkeit fördere, da er immer sehr ,hibbelig“ sei, sich aber in Mathe immer besonders konzentrieren müsse.

\subsection{Lehrererklärungen}

Die zweite Forschungsfrage der vorliegenden Studie zielte darauf ab zu beleuchten, welche Rolle Diskurse zu Lehrererklärungen in der Identitätsarbeit der Befragten im schülerzentrierten Unterricht spielen. Zwar wurden Lehrererklärungen in keiner Interviewfrage direkt adressiert. Gleichwohl zeigte die durchgeführte thematic analysis, dass Lehrererklärungen unter den Befragten das am häufigsten angesprochene Thema war. Fast alle Schüler und Schülerinnen beider Klassen, welche die Frage, ob sie mit ihrer Lehrperson zufrieden seien, nicht bejahten, gaben als häufigsten Grund dafür ihre Unzufriedenheit mit den Erklärungen ihrer Lehrperson an.

In Klasse 8, der Freiarbeitsklasse, gaben 19 Schüler und Schülerinnen (79\%) und in der Klasse 914 Schüler und Schülerinnen (67\%) an, dass neue Themen unter anderem mit Hilfe eines Lehrervortrages eingeführt werden. Neben der Selbsterarbeitung scheint also ein Lehrerinput in Form eines Vortrags auch gängig zu sein, obwohl die meisten Befragten bei den Erarbeitungsmethoden hauptsächlich von einer Selbsterarbeitung sprechen. Aber auch in einem schülerzentrierten offenen Unterricht sind Lehrererklärungen von Nöten, liegt doch nun die Hauptaufgabe der Lehrkraft in der individuellen Hilfestellung der einzelnen Lernenden. Diese neue Aufgabe wird auch von den Schülern und Schülerinnen als solche genannt: Aus der Klasse 8 antworteten 21 von 24 Befragten auf die Frage, welche Rolle die Lehrkraft im Unterricht spiele, dass diese für Fragen zur Verfügung stehe und bei Unklarheiten helfe. In der Klasse 9 gaben alle Befragten eine entsprechende Antwort.

Eine große Anzahl der Schüler und Schülerinnen aus beiden Klassen gab an, mit den Erklärungen der Lehrkräfte nicht zufrieden zu sein. Aus der Klasse 8 waren $54 \%$ unzufrieden mit den Erklärungen der Lehrkraft, in der Klasse 9 waren es $43 \%$ aller Schüler und Schülerinnen. Die Gründe hierfür waren verschiedene. In beiden Klassen gab ein Drittel der Schüler und Schülerinnen an, sich die Inhalte des Unterrichts lieber von ihren Mitschülern und Mitschülerinnen erklären zu lassen, da dies verständlicher sei. So sagte Marisa: ,wir haben auch Schüler, die einfach mir das besser erklären können und es ist einfach kompliziert und ich hab' auch manchmal Angst sie [die Lehrkraft] zu fragen, weil ich Angst habe, dass sie mich für ein biss- 
chen blöd hält"“. Der Hauptgrund für diese Negativbewertung der Lehrererklärungen aus der Klasse 9 war, dass diese nicht verstanden wurden. So meinte Annett:

Mit dem Erklären, das ist halt immer ziemlich anstrengend, weil es genauso erklärt wird, wie es am Anfang auch erklärt wurde, und dann verstehe ich das nicht. [...] Sie erklärt es dann genauso, wie sie es vorher auch erklärt hat, wo man es ja auch schon nicht verstanden hat. [...] Und dann fragt man halt immer eher die Nachbarin, weil das dann immer besser erklärt werden kann.

Einige Schüler und Schülerinnen erklärten, dass die Lehrererklärungen auf Grund von verwendeten Fachausdrücken nicht verstanden werden. Maria sagte dazu:

\begin{tabular}{ll}
\hline Maria: & Wenn ich nicht mehr weiterkomme, suche ich mir halt Hilfe, aber meistens mehr bei \\
& Mitschülern, weil die mir das auch besser erklären können [...] \\
Interviewerin: & Warum? \\
Maria: & Ich weiß es nicht, ich glaub einfach, dadurch, dass sie es sich selbst gerade eben erst \\
& {$[\ldots]$ erschlossen haben, [...] können sie es glaube ich besser erklären, können besser so } \\
& 'reinfühlen, was man vielleicht nicht versteht oder so \\
Interviewerin: & Wenn dir das deine Mitschüler erklären, reden die dann auch anders als die Lehrkraft? \\
& Also nutzen die auch andere Wörter? \\
Maria: & Ja, ich glaube sie reden einfach so einfacher und nehmen nicht so viele Fachbegriffe und \\
& so \\
\hline
\end{tabular}

Maria hatte große Probleme, die Erklärungen der Lehrkraft zu verstehen. Sie wandte sich deshalb an ihre Mitschüler und Mitschülerinnen, um sich von ihnen helfen zu lassen. Dasselbe berichteten auch neun weitere Lernende. Ein wichtiger Punkt, der von Maria genannt wurde, ist die Verwendung von Fachausdrücken. Mit diesem Problem war sie nicht allein; so gab es mehrere Schüler und Schülerinnen, die angaben, dass die Lehrererklärungen auf Grund der Verwendung von Fachtermini nicht verstanden und so auch nicht nachvollzogen werden können.

Ein weiterer wichtiger Faktor, weshalb Unzufriedenheit gegenüber den Lehrererklärungen entstand, war die Zeit. So bemängelten $13 \%$ der Schüler und Schülerinnen der Klasse 8, dass die Lehrerin auch manchmal nicht erklären möchte, was allerdings noch eine didaktische Entscheidung darstellen könnte. Jedoch berichteten auch $29 \%$ der Befragten, dass durch die Freiarbeit und die damit verbundenen individuellen Fragen einzelner Schüler und Schülerinnen für die Lehrkraft oft nicht die Zeit bleibe, alle Fragen zu beantworten und alle Unklarheiten aus dem Weg zu räumen. Milan, ein Schüler aus Klasse 8, sagte, dass ,manchmal auch so viele Fragen aufkommen, dass sie's gar nicht schafft“. Fast die Hälfte aller Schüler und Schülerinnen beider Klassen wünschte sich mehr Frontalunterricht in den in beiden Fällen sehr schülerzentrierten Unterricht einzubauen.

Auch wenn der schülerzentrierte Unterricht neben den Lehrererklärungen vielfältige Zugänge zu mathematischem Verständnis bot, schienen die Qualität und Quantität der Lehrererklärungen einen essentiellen Anteil am Lernerfolg zu haben: So gaben aus der Klasse $825 \%$ der Befragten und aus der Klasse $919 \%$ der Befragten im Gespräch ohne gezielte Frage danach an, dass ihr Verständnis von den Lehrererklärungen abhänge. So antwortete Elena aus Klasse 9 auf die Frage, ob sie Mathe verständlich finde: „Für mich kommt es immer darauf an, wer es mir erklärt, 
wie es verständlich dann für mich ist, weil, es gibt manche Personen, die können's mir relativ einfach erklären und mache Personen, da versteh' ich dann nur Bahnhof". Und auch Charlotte aus Klasse 8 erläuterte, dass die Lehrkraft die Sachverhalte zwar erkläre, sie aber trotz dieser Erklärung kaum etwas verstehe. Das sagte auch Karl, der nicht nur die Erklärfähigkeit der Lehrkraft mit seinem Verständnis in Verbindung brachte, sondern hinzufügte, dass er durch sein meist sehr spätes Verständnis sehr unzufrieden mit der Lehrkraft sei.

Dennoch verfügten große Teile der Schüler und Schülerinnen aus beiden Klassen ihren Aussagen zufolge über Alternativen, welche ihnen bei Verständnisproblemen helfen. Sie nannten hier neben der Lehrkraft nicht nur Familienangehörige oder Mitschüler und Mitschülerinnen; auch die den Schülern und Schülerinnen zur Verfügung gestellten Lernvideos dienten als Unterstützung. So antwortete beispielsweise Mija auf die Frage, was ihr helfe, wenn sie Probleme in Mathe habe: ,[...] ich guck mir im Internet Videos an, ich habe auch eine App. Oder ich frage meine Mitschüler oder rede mit meinem Papa, weil der mich auch ganz gut motivieren kann." Und auch Karl sucht sich Hilfe bei seinen Mitschülern und Mitschülerinnen und argumentiert, dass ,die [es] einem dann gut erklären [können]. Und wenn das nicht der Fall ist, dann versuche ich mir das mit Hilfe des Internets oder meinen Eltern zu erklären.“ Diese Alternativen machten es möglich, aus der Rolle des oder der passiven Lernenden, der oder die an das Wissen der Lehrkraft gebunden ist, in eine aktivere Rolle zu wechseln, in welcher die Abhängigkeit von der Lehrkraft vermindert wird, welche einen gewissen Freiraum für selbstreguliertes Lernen einräumt und zugleich die Rolle der Lehrkraft im Lernprozess verändert.

\subsection{Persönlichkeitseinbringung}

Die Schüler und Schülerinnen wurden gefragt, ob es ihnen möglich sei, im Unterricht ihre Persönlichkeit einzubringen. Viele Befragte fanden die Frage zunächst unverständlich, so dass sie umformuliert erneut gestellt wurde oder am Beispiel der Auslebung der eigenen Kreativität eine exemplarische Antwortdimension aufgezeigt wurde. Die Befragten konnten dahingehend eigene Gedanken beitragen. Schließlich gaben $90 \%$ der Klasse 8 und $66 \%$ der Klasse 9 an, ihre Persönlichkeit auch im Unterricht einbringen zu können.

Hier ist deutlich erkennbar, dass der Teil der Schüler und Schülerinnen, die in der Klasse 8 der Auffassung waren, ihre Persönlichkeit im Unterricht einbringen zu können, deutlich über dem der aus Klasse 9 lag. Aus den Schülerantworten lässt sich ableiten, dass hierfür vermutlich die zusätzlichen Möglichkeiten zur Leistungserbringung, welche im Mathematiika-Konzept vorgesehen sind, verantwortlich sind, etwa die Möglichkeiten für eine Zusätzliche Leistungsbeurteilung (ZuLB), die gerne für die Erstellung kreativer Lernprodukte genutzt werden. Beispielhaft sei dies durch Marisa aus der Klasse 8 erläutert: „Ja, es gibt halt nach jedem Modul, was wir sozusagen haben, ein ZuLB, was wir machen dürfen, also zum Beispiel so 'n Spiel erstellen und das ist halt sowas, wo man halt endlich kreativ werden kann, so. [...] Das find ich cool, ja.“

Vergleichend dazu antwortete Rainer aus Klasse 9 auf die Frage, ob er im Unterricht eigene Ideen oder Vorschläge einbringen könne: „Eher nicht, weil man halt 'ne 
feste Aufgabe hat, die man lösen muss und, ja, es gibt eigentlich wenige Lösungswege und da kann man eigentlich nicht wirklich Kreativität einbringen“. Charlotte aus Klasse 8 war zunächst der gleichen Auffassung und argumentierte, dass ihr Lieblingsfach Kunst sei, da man in diesem Fach kreativ sein könne. Sie verneinte zunächst die Frage, ob man seine Persönlichkeit im Mathematikunterricht einbringen könne, ergänzte aber auf Nachfragen, dass man im Rahmen der ZuLBs, welche sie als „etwas Kreatives“ beschrieb, auch sehr kreativ sein könne und diese auch Raum für eigene Ideen ließen. Ähnlich in Bezug auf die in der Mathematik angewendete Kreativität äußerte sich Emilyan. Dieser gab an, dass seine Lieblingsfächer Musik und Kunst seien, weil er ,gerne kreative Sachen mache, das ist meine Stärke. Deswegen find ich solche kreativen Fächer einfach gut, macht mir Spaß, bin ich gut drin“. Er unterschied diese Fächer ausdrücklich von der Mathematik und meinte, dass es kreative und nicht kreative Fächer gebe. Er wollte zwar nicht ausschließen, dass Mathematik nicht auch Raum für Kreativität lasse, war aber der Auffassung, dass es in der Mathematik eher nicht möglich sei, seine Kreativität und in diesem Zusammenhang auch seine Persönlichkeit einzubringen.

Spezifischer als allgemeine Verweise auf fehlende Möglichkeiten, kreativ zu werden, waren die Aussagen einiger Schüler und Schülerinnen, dass es in Mathe nur richtig oder falsch gebe, was eng mit der logischen Dimension von Mathe verknüpft wurde. So argumentierte Charlotte aus Klasse 8, dass Kunst ihr Lieblingsfach sei, denn „Kunst ist halt, ja so kreativ und es gibt, sag ich, nicht so richtig und falsch, sondern man kann einfach zeichnen“. Es soll jedoch nicht unerwähnt bleiben, dass die logische Dimension der Mathematik von einigen Schülern und Schülerinnen auch durchaus positiv bewertet wurde. So erklärte Lenia aus Klasse 8, dass sie bezüglich der Mathematik schätze, ,,[d]ass es logisch ist, also das ist so bei Kunst zum Beispiel, ich weiß immer nicht, ob ich was richtig mache oder nicht, weil das hat ja keine Logik“.

Einige Schüler und Schülerinnen sahen Mathematik als eine regelgeleitete Aktivität an, die kaum Raum für Persönlichkeitseinbringung lässt. Eine von diesen Schülern und Schülerinnen war Marisa aus Klasse 8, die wie folgt argumentierte: ,in der Mathematik gibt's immer nur so 'n ja, es ist richtig oder es ist falsch und sowas find ich manchmal ein bisschen blöd, weil so eigene Meinung kaum gefragt wird und das ist halt in ander'n Fächern nicht so“. Im weiteren Gespräch merkte Marisa an, dass es in Mathematik weniger um Argumentationen und einen eigenen Beitrag gehe, vielmehr müsse man die Aufgabe einfach richtig beantwortet haben. Es gebe ihrer Meinung nach eben nur richtig und falsch. Auch Elena aus Klasse 9 stimmte dieser Meinung zu; sie sagte, dass es in der Mathematik eher darum gehe, immer die Aufgabe nach demselben Schema zu lösen, man habe keine „Ausweichmöglichkeiten“" in der Bearbeitung.

Alvin aus Klasse 8 war hingegen eher der Auffassung, dass es in der Mathematik nur ,richtig“ gebe, denn ,,jede Denkweise ist an sich ein richtiger Schritt. [...] [D]eswegen würde ich nicht sagen, dass es ein Falsch gibt, weil jede Überlegung richtig ist." Diese Aussage lässt erkennen, dass es auch Schüler und Schülerinnen gab, welche den Lösungsweg und die damit verbundenen eigenen Gedanken bereits als wichtigen Beitrag zum Unterricht ansehen. So gab neben Alvin ein Großteil der Schüler und Schülerinnen beider Klassen an, dass sie Mathematik eher mit einem 
vielseitigen Lösungsverständnis in Verbindung bringen. Einer von ihnen war Martin aus Klasse 8, der argumentierte, dass ,'ne Aufgabe [...] ja oft richtig gerechnet werden [muss], aber es [...] oft verschiedene Lösungswege [gibt] und da kann man jetzt nicht sagen, ja der Lösungsweg ist jetzt komplett falsch, wenn er zur [richtigen] Lösung führt“. Und auch Noah aus Klasse 9 bestätigte die verschiedenen Lösungsmöglichkeiten mit der Aussage: „Denn es gibt immer Alternativen“. Mija aus Klasse 9 meinte, man könne seine Persönlichkeit durchaus im Mathematikunterricht einbringen, ,weil jeder hat ja so eigene Möglichkeiten oder eigene Ideen, Sachen zu lösen oder an Sachen ranzugehen, an neue Aufgaben und deswegen, denke ich, unterscheidet sich das auch, also viele Wege führen ja zum Ziel und deswegen kann jeder, so wie er halt arbeitet zu seiner Lösung finden“.

\section{Diskussion und Ausblick}

Die erste Forschungsfrage, also inwieweit sich die Schüler und Schülerinnen durch das gezielte Fragen nach Relevanzzuschreibungen jenseits des Lernens von Mathematik in weiteren Diskursen zur Relevanz von Mathematik verorten, konnte erfolgreich beleuchtet werden. Zunächst zeigte sich das verknappte Antwortverhalten auf die Frage, wozu Mathematik denn wichtig sei, wie es bereits in den Studien von Maaß und Ege (2007), Kollosche (2017a) und Sachdeva und Eggen (2019) dokumentiert wurde. Der Rückfall auf Diskurse, die die Nützlichkeit elementarer mathematischer Inhalte betonen, das Unvermögen, die behauptete Nützlichkeit der Mathematik mit Beispielen aus dem weiterführenden Mathematikunterricht zu unterlegen, aber auch Signalwörter wie ,eigentlich“ (Anita, Ilyas) und ,bestimmt“ (Ilyas), die mehr ein Hoffen als eine Überzeugung ausdrücken mögen, zeugen auch hier von der Fragilität der vorgetragenen Relevanzzuschreibungen. Hervorzuheben ist an dieser Stelle die Äußerung von Ilyas, dass die herausgestellte Wichtigkeit von Mathematik als großer Druck auf ihm laste. Dies deutet zumindest an, dass womöglich übertriebene Erwartungen an das im späteren Leben benötigte mathematische Wissen und Können dem Mathematiklernen eine Bedeutungsschwere verleihen kann, die der Entwicklung einer lernförderlichen Identität als Mathematiklernende im Wege steht. Insgesamt bleiben die vorgebrachten Diskurse, wozu Mathematik wichtig sei, hier verknappt und fragil und beschränken damit maßgeblich, inwieweit Mathematik als ein Gegenstand begriffen werden kann, der von Schülern und Schülerinnen im Diskurs über das Selbst als passend zu eigenen Bestrebungen verortet werden kann.

Gezieltes Nachfragen, insbesondere mit der Frage „Lernst du in Mathe etwas für dich, das über die Lerninhalte hinausgeht?“, brachte dann aber seitens der Befragten weitere Diskurse zur Relevanz von Mathematik zur Sprache. Der häufige Erfolg des gezielten Nachfragens weist darauf hin, dass Schüler und Schülerinnen durchaus mehr als bisher beobachtet zum Thema zu sagen haben. Dafür spricht auch, dass Vorhölter (2009) und Vollstedt (2011) in ihren theoretisch anders gerahmten Interviewstudien zur Sinnkonstruktion von Schülerinnen und Schülern im Mathematikunterricht durchaus reichhaltige Diskurse rekonstruieren konnten. Warum es in der vorliegenden Studie notwendig war, auf dem Thema zu insistieren, lässt sich 
hier nur vermuten. Zum einen mag es sein, dass die Befragten hierzu noch kaum Position bezogen haben und während der Befragung überhaupt erst eine erste Identitätsarbeit einsetzt. Zum anderen deuten die Daten darauf hin, dass der Diskurs, in dem die Bedeutung von Mathematik in der lebensweltlichen Anwendung der erlernten Inhalte verortet wird, an die Schüler und Schülerinnen herangetragen und von ihnen nur unkritisch reproduziert wird. Die damit kultivierte Standardantwort mag einer gehaltvollen Auseinandersetzung mit der Frage dann im Wege stehen. Zudem bleibt unklar, inwieweit Selektionseffekte durch den Status als Begabungsklassen das Auftreten elaborierter Diskurse zur Relevanz von Mathematik begünstigt haben. Gleichwohl nähren die Erkenntnisse die Hoffnung, dass ein Gespräch zu Sinn und Zweck von Mathematik und Mathematikunterricht möglich ist, den Schülern und Schülerinnen in ihrer Identitätsarbeit im Mathematikunterricht unterstützen mag und durch eine entsprechende Lernumgebung angeregt werden kann. Kollosche (2019b) schlägt hierzu eine unterrichtliche Umsetzung vor.

Die nachgeschobene Frage zu über die Lerninhalte Hinausgehendes führte zu einer deutlich größeren Anzahl von Antworten in der Freiarbeitsklasse. Die Analyse der Diskurse der Befragten lässt darauf schließen, dass diese Unterschiede im Ausbilden von Fähigkeiten zur Selbstführung zu finden sind. Es kann vermutet werden, dass hierzu durch die nötigen Strategiebesprechungen im Freiarbeitsunterricht auch ein stärkeres Bewusstsein unter den Schülern und Schülerinnen geschaffen wurde. Damit legen die Diskurse der Befragten nahe, dass stark selbstorganisiertes Lernen die Vielfalt dessen, was im Mathematikunterricht gelernt werden kann, bereichert. Man könnte nun fragen, inwieweit diese Relevanzzuschreibungen mit dem Fach Mathematik oder unabhängig vom Fach mit der Unterrichtsform allgemein in Beziehung stehen. Wenn Ursula von dem Erlernen des selbständigen Arbeitens oder Emilyan von der Förderung seiner Konzentrationsfähigkeit in der Freiarbeit spricht, werden ausschließlich Charakteristika der Unterrichtsform angesprochen. Zwar konstituieren auch solche Charakteristika des Mathematikunterrichts den Gegenstand Mathematik, hier etwa nicht als Sammlung von mitgeteilten Fakten und imitierten Praktiken, sondern als eigenaktive und charakterbildende intellektuelle Tätigkeit, doch diese Lernerfahrung wäre unabhängig von der Mathematik auch in anderen Fächern möglich. Fachspezifische, inhaltsübergreifende Charakteristika, wie etwa das logisch-deduktive Argumentieren oder das mathematische Modellieren, werden von den Befragten hingegen nicht für Relevanzzuschreibungen herangezogen. Offenbar werden insbesondere die in den deutschen Bildungsstandards benannten ,allgemeinen mathematischen Kompetenzen“ (bspw. KMK 2015, S. 11) von den Lernenden nicht als Möglichkeiten für Relevanzzuschreibungen erkannt.

Hinsichtlich der zweiten Forschungsfrage, also welche Rolle Diskurse zur Qualität von Lehrererklärungen in der Identitätsarbeit im schülerzentrierten Unterricht spielen, zeigte sich zunächst, dass auch in den hier untersuchten schülerzentrierten Klassen die Qualität der Lehrererklärungen das von den Befragten am häufigsten adressierte Thema darstellte. Damit wurden recht eindrucksvoll frühere Ergebnisse in Klassen mit lehrerzentriertem Unterricht reproduziert (Kollosche 2017b). Dies überrascht, da man hätte erwarten können, dass durch die schülerzentrierte Organisation des Lernens in den hier untersuchten Klassen die Lehrererklärungen keine zentrale Rolle in den Diskursen der Befragten spielen. Eine mögliche Erklärung 
der Persistenz des Themas ist, dass durch die Sozialisierung im früheren Mathematikunterricht und durch die anhaltende Sozialisierung im anderen Fachunterricht Verhaltensmuster des lehrerzentrierten Unterrichts eingeprägt wurden und den Erklärungen der Lehrpersonen daher mit großen Erwartungen begegnet wurde. Auch mag es sein, dass Lehrererklärungen vielen Befragten trotz der vorgetragenen Vorbehalte als verlockende Alternative zu einer mühsameren eigenen Erarbeitung erscheinen. $\mathrm{Zu}$ beiden Erklärungen würde passen, dass sich fast die Hälfte der Schüler und Schülerinnen beider Klassen mehr Frontalunterricht wünschte. Andererseits scheint es nur natürlich, sich von fachlichen Experten und Expertinnen hilfreiche Erklärungen zu erhoffen. Dass die Erklärung dann oft als nicht hilfreich empfunden werden, kann viele Ursachen haben, etwa didaktisch unzureichende Erklärungen seitens der Lehrperson oder unterschiedliche Diskurs- und Erfahrungswelten von Lernenden und Lehrenden. Eine gewisse Spannung zwischen erwünschten und dargebotenen Erklärungen mag letztlich auch wünschenswert sein, insofern die Lehrperson den Lernenden ein noch fremdes mathematisches Denken und Sprechen vorführen und kultivieren soll.

Auffällig war hinsichtlich des hier untersuchten schülerzentrierten Unterrichts, dass nur wenige Schüler und Schülerinnen dieser Klassen unzureichende Lehrererklärungen für ihre eigenen Misserfolge verantwortlich machten. Zum einen zeigten einige Schüler stattdessen ein Bewusstsein für die begrenzten zeitlichen Ressourcen der Lehrperson, die ihr nicht erlauben, im schülerzentrierten Unterricht jedem individuelle Erklärungen anzubieten. Zum anderen verwiesen viele Schüler und Schülerinnen auf alternative Lernquellen wie Mitschüler und Mitschülerinnen oder Lernvideos. Diese Vielfalt impliziert, dass Schüler und Schülerinnen - anders als im von Kollosche (2017b) untersuchten lehrerzentrierten Unterricht - eigenen Misserfolg in ihrer Identitätsarbeit nicht mehr monokausal auf unzureichende Lehrererklärungen zurückführen können, sondern sich tiefergehend mit dessen Ursachen befassen müssen.

Zur dritten Forschungsfrage, also wie die Befragten mit schülerzentriertem Unterricht ihre Möglichkeiten zur Persönlichkeitseinbringung bewerten, lassen sich mehrere Erkenntnisse festhalten. Zunächst sehen einige Schüler und Schülerinnen ihre Möglichkeiten zur Persönlichkeitseinbringung im Mathematikunterricht auf Grund der in der Mathematik vorherrschenden logischen Determiniertheit von Aussagen und der Regelhaftigkeit von Verfahren als begrenzt an. Hiermit wird ein Diskurs reproduziert, den Boaler und Greeno (2000) bereits in ihrer Untersuchung von lehrerzentriertem Unterricht beschrieben hatten. Dass entsprechende Diskurse anders als bei Boaler und Greeno hier auch im schülerzentrierten Unterricht auftauchen, überrascht. Mögliche Erklärungen wären, dass es sich hier um notwendige Charakteristika von Mathematik handelt, die auch durch alternative Unterrichtsformen nicht hintergehbar sind, dass der in den untersuchten Klassen umgesetzte Unterricht trotz schülerzentrierter Arbeitsformen noch vorwiegend auf wissens- und regelorientierte Lerninhalte setzte, oder dass bei einigen Schülern und Schülerinnen noch Eindrücke auf früherem, lehrerzentriertem Unterricht nachwirken. Welche dieser Erklärungen belastbar ist, lässt sich auf der Grundlage des vorliegenden Datenmaterials nicht klären. Gegen die zweite Erklärung spricht aber, dass Schüler wie Martin aus Klasse 8 die Bedeutung verschiedener Lösungswege betont. Bemerkenswert ist 
an dieser Stelle auch die Einschätzung der Lehrkraft von Klasse 8, dass die meisten Schüler und Schülerinnen als Zugangsformen die Bibliothek und die Mediathek und damit rezeptive Zugangsformen wählten und das problemorientierte Arbeiten mit den Inhalten der Safari mieden.

Im Gegensatz zu den im letzten Absatz diskutierten Vorbehalten einiger Schüler und Schülerinnen ist es jedoch erfreulich, dass $90 \%$ der Schüler und Schülerinnen in Klasse 8 und $66 \%$ in Klasse 9 sagten, dass sie ihre Persönlichkeit im Mathematikunterricht einbringen könnten. Wir führen diese hohen Werte auf die schülerzentrierte Organisation des untersuchten Unterrichts zurück, womit ein zentrales Ergebnis der Studie von Boaler und Grenno (2000) hier reproduziert werden konnte. Die im Vergleich zwischen beiden Klassen deutlich höhere Zustimmung in der Mathematiika-Klasse lässt sich zumindest teilweise zurückführen auf die Möglichkeiten, welche die Zusätzliche Leistungsbeurteilung in dieser Klasse bot. Inwieweit die kreative Auseinandersetzung mit Mathematik hier noch lehrplanorientiert und ein fester Bestandteil des Unterrichts oder aber eine weitgehend von Unterrichtsinhalten losgelöste Aktivität darstellte, ist auf der Grundlage unserer Daten nicht zu beantworten. Ebenfalls unklar ist, inwieweit die Möglichkeit zur kreativen Auseinandersetzung mit Mathematik den Schülern und Schülerinnen auch Möglichkeiten zur Persönlichkeitseinbringung im alltäglichen Unterricht eröffnet hat.

Indes deuteten die Schwierigkeiten der Befragten, auf die Frage nach der Möglichkeit des Einbringens ihrer Persönlichkeit in den Mathematikunterricht überhaupt $\mathrm{zu}$ antworten, darauf hin, dass zuweilen gar keine Sprache kultiviert ist, um über die Beziehung des Selbst zum Mathematikunterricht zu reflektieren. Indem in solchen Fragen, Antwortversuchen und Praktiken des Nachfragens erst gegenseitig verständliche Diskurse entwickelt werden, gerät die Interviewsituation selbst notwendig zu einem Ort von Identitätsarbeit unter Einfluss der Interviewenden. Dabei stellt sich durchaus die Frage, inwieweit das kommunikative Handeln der Interviewenden die Diskurse der Befragten lenken, etwa wenn die Frage nach der Persönlichkeitseinbringung bei Bedarf am Beispiel des Einbringens der eigenen Kreativität und nicht an anderen Beispielen konkretisiert wird. Der Mangel an für Schüler und Schülerinnen verfügbaren Diskursen zum Sprechen über Mathematikunterricht kann dabei durchaus als Hürde aufgefasst werden, die die Artikulation von lernförderlichen Identitäten, aber auch ihre wissenschaftliche Untersuchung erschwert.

Es sollte daran erinnert werden, dass die beiden untersuchten Klassen singuläre Lernorte darstellen und vermutlich nicht als repräsentative Manifestation von schülerzentriertem Unterricht aufgefasst werden können, und dass Besonderheiten in den präsentierten Ergebnissen mit dem Status als Begabungsklassen in Zusammenhang stehen können - auch wenn die Befragten in ihren Aussagen an keiner Stelle einen Zusammenhang zu diesem Status herstellten. Gleichwohl lässt sich auf der Grundlage der vorliegenden Studie festhalten, dass Schüler und Schülerinnen unhinterfragte Relevanzzuschreibungen durchaus überwinden und zu einem reichhaltigen Diskurs über die Relevanz von Mathematikunterricht vordringen können. Auch scheint es möglich, durch schüler- statt lehrerzentrierten Unterricht die empfundene Abhängigkeit von Lehrererklärungen zu vermindern und die Möglichkeiten zur Persönlichkeitseinbringung auszubauen. 
Die Äußerungen der befragten Schüler und Schülerinnen zeigten dabei deutlich, dass ihre Identitätsarbeit im Mathematikunterricht eng mit den jeweils vorherrschenden Parametern der Unterrichtsgestaltung verflochten war. Dieser Befund bestätigt einmal mehr, dass es möglich und sinnvoll ist, die Auswirkungen der Unterrichtsgestaltung auf die Identitäten von Mathematiklernenden in den Blick zu nehmen. In diesem Beitrag konnten zu diesem Zweck nur ausgewählte Fragestellung an einer außergewöhnlichen Schülergruppe diskutiert werden. Allein dabei warf die hier präsentierte Studie bedeutsame Fragen auf, etwa wie reichhaltige Relevanzzuschreibungen, auch bezüglich prozessbezogener mathematischer Kompetenzen, initiiert werden können und wie sich diese auf Identitätsarbeit auswirken, oder wie sich erklären lässt, warum Lehrererklärungen auch in hier untersuchten, schülerzentriert unterrichteten Klassen ein zentrales Thema der Diskurse der Befragten zum Mathematikunterricht darstellen. Für ein tieferes Verständnis, wie die Gestaltung von Mathematikunterricht und die Identität von Mathematiklernenden zusammenwirken und welche Auswirkungen dieses Zusammenwirken auf das Lernen und das Mündigwerden im Mathematikunterricht hat, werden weitere Forschungsanstrengungen nötig sein.

Zusatzmaterial online Zusätzliche Informationen sind in der Online-Version dieses Artikels (https://doi. org/10.1007/s13138-021-00187-2) enthalten.

Danksagung Wir danken Lena Florian für ihren Rat bei der Planung der Studie und ihre Hilfe beim Verfassen des ersten Entwurfs dieses Beitrags. Wir danken ferner den anonymen Gutachtern und Gutachterinnen des Beitrags für die zahlreichen Hinweise, die zur Verbesserung des Beitrags beitrugen.

Funding Open access funding provided by University of Klagenfurt.

Open Access Dieser Artikel wird unter der Creative Commons Namensnennung 4.0 International Lizenz veröffentlicht, welche die Nutzung, Vervielfältigung, Bearbeitung, Verbreitung und Wiedergabe in jeglichem Medium und Format erlaubt, sofern Sie den/die ursprünglichen Autor(en) und die Quelle ordnungsgemäß nennen, einen Link zur Creative Commons Lizenz beifügen und angeben, ob Änderungen vorgenommen wurden.

Die in diesem Artikel enthaltenen Bilder und sonstiges Drittmaterial unterliegen ebenfalls der genannten Creative Commons Lizenz, sofern sich aus der Abbildungslegende nichts anderes ergibt. Sofern das betreffende Material nicht unter der genannten Creative Commons Lizenz steht und die betreffende Handlung nicht nach gesetzlichen Vorschriften erlaubt ist, ist für die oben aufgeführten Weiterverwendungen des Materials die Einwilligung des jeweiligen Rechteinhabers einzuholen.

Weitere Details zur Lizenz entnehmen Sie bitte der Lizenzinformation auf http://creativecommons.org/ licenses/by/4.0/deed.de.

\section{Literatur}

Andersson, A., Valero, P., \& Meaney, T. (2015). "I am [not always] a maths hater": Shifting students' identity narratives in context. Educational Studies in Mathematics, 90(2), 143-161.

Boaler, J., \& Greeno, J.G. (2000). Identity, agency, and knowing in mathematics worlds. In J. Boaler (Hrsg.), Multiple perspectives on mathematics teaching and learning (S. 171-200). Westport: Ablex.

Boaler, J., William, D., \& Zevenbergen, R. (2000). The construction of identity in secondary mathematics education. In J.F. Matos \& M. Santos (Hrsg.), Proceedings of the 2nd International Mathematics Education and Society Conference (S. 192-202). Montechoro: Universidade de Lisboa.

Braun, V., \& Clarke, V. (2006). Using thematic analysis in psychology. Qualitative Research in Psychology, $3(2), 77-101$ 
Chronaki, A., \& Kollosche, D. (2019). Refusing mathematics: A discourse theory approach on the politics of identity work. ZDM Mathematics Education, 51(3), 457-468.

Darragh, L. (2016). Identity research in mathematics education. Educational Studies in Mathematics, 93(1), 19-33.

Flick, U. (2000). Qualitative Forschung: Theorie, Methoden, Anwendung in Psychologie und Sozialwissenschaften. Reinbek bei Hamburg: Rowohlt.

Florian, L., \& Grabow, S. (2017). mathe matiika [YouTube-Kanal]. https://www.youtube.com/c/ mathematiika. Zugegriffen: 22. Juni 2021.

Foucault, M. (1975). Surveiller et punir: Naissance de la prison. Paris: Gallimard.

Foucault, M. (1981). Archäologie des Wissens. Frankfurt am Main: Suhrkamp. Erstveröffentlichung 1969 als L'archéologie du savoir

de Freitas, E. (2008). Mathematics and its other: (Dis)locating the feminine. Gender and Education, 20(3), $281-290$.

Graven, M., \& Heyd-Metzuyanim, E. (2019). Mathematics identity research: The state of the art and future directions. ZDM Mathematics Education, 51(3), 361-377.

Heymann, H. W. (1996). Allgemeinbildung und Mathematik. Weinheim: Beltz.

Ingram, N. (2011). Affect and identity: The mathematical journeys of adolescents. Dunedin: University of Otago.

KMK (2015). Bildungsstandards im Fach Mathematik für die Allgemeine Hochschulreife. Köln: Wolters Kluwer. http://www.kmk.org/fileadmin/Dateien/veroeffentlichungen_beschluesse/2012/2012_10_ 18-Bildungsstandards-Mathe-Abi.pdf

Kollosche, D. (2017a). The ideology of relevance in school mathematics. In A. Chronaki (Hrsg.), Mathematics education and life at times of crisis (S. 633-644). Volos: University of Thessaly Press.

Kollosche, D. (2017b). The socio-politics of teacher explanation in mathematics education. In T. Dooley \& G. Gueudet (Hrsg.), Proceedings of the Tenth Congress of the European Society for Research in Mathematics Education (S. 1505-1512). Dublin: DCU Institute of Education.

Kollosche, D. (2019a). Reasons for auto-exclusion: Why students reject mathematics. In D. Kollosche, R. Marcone, M. Knigge, M. G. Penteado \& O. Skovsmose (Hrsg.), Inclusive mathematics education: State-of-the-art research from Brazil and Germany (S. 449-465). Cham: Springer.

Kollosche, D. (2019b). Wozu Mathematik lernen? Ziele besprechen und verinnerlichen. Der Mathematikunterricht, 65(5), 48-56.

Laclau, E., \& Mouffe, C. (1985). Hegemony and socialist strategy: Towards a radical democratic politics. London: Verso.

Maßß, K., \& Ege, P. (2007). Mathematik und Mathematikunterricht aus der Sicht von Hauptschülern. mathematica didactica, 30(2), 53-85.

Mendick, H. (2005). A beautiful myth? The gendering of being/doing "good at maths". Gender and Education, 17(2), 203-219.

Mendick, H. (2006). Masculinities in mathematics. Maidenhead: Open University.

Radovic, D., Black, L., Williams, J., \& Salas, C.E. (2018). Towards conceptual coherence in the research on mathematics learner identity: A systematic review of the literature. Educational Studies in Mathematics, 99(1), 21-42.

Sachdeva, S., \& Eggen, P.-O. (2019). Students' critical perceptions about mathematics education. In J. Subramanian (Hrsg.), Proceedings of the Tenth International Mathematics Education and Society Conference (S. 761-770). Hyderabad: MES 10.

Vollstedt, M. (2011). Sinnkonstruktion und Mathematiklernen in Deutschland und Hongkong: Eine rekonstruktiv-empirische Studie. Wiesbaden: Vieweg + Teubner.

Vorhölter, K. (2009). Sinn im Mathematikunterricht: Zur Rolle von mathematischen Modellierungsaufgaben bei der Sinnkonstruktion von Schülerinnen und Schülern. Leverkusen: Budrich. 